\title{
Profiles
}

\section{Liubo Zhang, China CDC's Chief Expert of Disinfection}

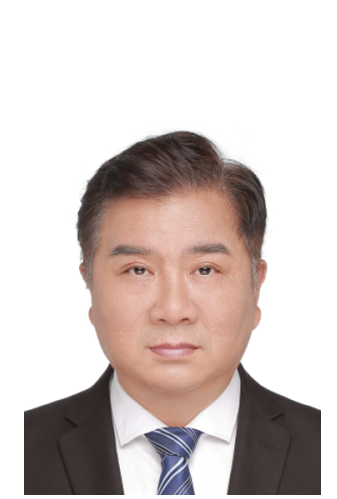

\author{
Peter Hao ${ }^{1, \alpha} ;$ Yu Chen ${ }^{2, x} ;$ Zhenjun $\mathrm{Li}^{1}$; Jingjing $\mathrm{Xi}^{1, * *} ;$ Feng Tan ${ }^{1, *}$
}

disease 2019 (COVID-19), etc. He was responsible for the development of disinfection technical guidelines and on-site guidance for these diseases. He also participated in formulating on-site guidance of technical guidelines for the disinfection of major natural disasters such as the Wenchuan Earthquake, Zhouqu Mountain's landslides, and Mozhu Mountain's landslides in Xizang (Tibet) Autonomous Region. He has been awarded the titles of the "New Long March Assaulter of the Ministry of Health", "Advanced Workers of the Ministry of Health", and Merit Citation Class III of Hubei Province.

His work has not only been on the frontlines of domestic disease prevention and control but also on the international stage for rescues and epidemic prevention and control. In 2005, as a member of the Chinese Emergency Medical Team, he went to Indonesia to carry out post-disaster rescue abroad and was responsible for environmental disinfection and health protection of medical team members. In 2014, he went to Sierra Leone in West Africa as the chief disinfection expert to carry out Ebola hemorrhagic fever prevention and control work. Zhang used comprehensive and excellent professional technology to contribute to the prevention and control of the international epidemic and fulfilled his commitment to serve the people of Sierra Leone and to fulfill the national mission.

During the COVID-19 pandemic, he led his team in the prompt development of more than 50 personal protection and scientific disinfection technical guidelines, 13 of which were adopted and officially released by the State Council's Joint Prevention and Control Mechanism or the National Health Commission (NHC, formerly the Ministry of Health). Zhang provided an expert's perspective through informing decisions and technical advice. He provided assistance to the State Council, the NHC, the Ministry of Industry and Information Technology, the Ministry of Public Security, the Ministry of Environmental Protection, the Civil Aviation Administration of China, and the National Railway Administration to solve problems such as the shortage of protective clothing and masks, hospital sewage treatment, protection and disinfection of special places and special populations, and the optimal use of disinfectants.

His proposals of "disposable protective clothing in isolation wards, excluding ICU isolation wards, should not consider microbial indicators" and the emergency marketing of some disinfection products were adopted and effectively alleviated the shortage of protective and disinfection materials in the early stages of the pandemic. Facing the phenomenon of excess disinfection, he issued a scientific disinfection proposal to promote the concept of "precision disinfection". He was interviewed by authoritative media and the Press Conference of the Joint Prevention and Control Mechanism of the State Council to publicize and popularize the scientific and precise prevention and control of COVID-19. Especially in the response to COVID-19 in Beijing, Liubo Zhang, as a member of the NHC's expert group and the Chief Expert of the Beijing Municipal Government's working group in Fengtai District, worked on-site for one month. He focused on guiding the on-site environmental cleaning, proper disposal, evaluation of the effects of disinfection, etc. At the city's regular meeting on COVID-19 prevention and control, he was praised by the leaders of the Beijing Municipal Government four times and was fully affirmed by the leaders of the NHC for his outstanding performance. 
The national hospital disinfection and infection control monitoring system he established has been in operation for more than 10 years and currently covers 32 provinces, cities, districts, and cadres across the country. It has accumulated a large amount of data for the prevention and control of infectious diseases and hospital infection control and promoted the development of hospital disinfection and infection control. As the first person in charge or sub-project leader, Zhang has presided over more than 10 national, provincial, and ministerial research projects and more than 10 special projects on disease control. The method "carrier flow immersion disinfection test" he studied was listed as one of the basic methods in the "Disinfection Technical Specification (2002 edition)". He led his team to a lot of breakthroughs, including the pressure steam sterilizer Bowie-Dick test (BD) and microbial resistance test system, and the hydrogen peroxide plasma sterilizer microbial resistance test device, the first establishment of cleaning effect evaluation laboratory for medical cleaning agents in China. Zhang led the drafting of 9 national standards, worked as 1 of 3 major individuals in charge of drafting 28 national standards and hygiene industry standards, and participated in the drafting of 21 national standards. He published more than 10 books on disinfection as Editor-in-Chief or Deputy Editor-in-Chief and published more than 50 articles in domestic and foreign magazines as the first author or corresponding author.

Liubo Zhang, as the Chief Expert of Disinfection, always promptly arrives at the scene of an epidemic or disaster to conduct on-site guidance and the development of disinfection technical guidelines. Wherever there is a catastrophe or an epidemic, he is there. He is a disease control fighter who is active on the front line of guarding the lives and health of the people and is also a leader in guiding the rapid development of disinfection in China. doi: $10.46234 / \mathrm{ccdcw} 2020.193$

\# Corresponding authors: Jingjing Xi, xijj@chinacdc.cn; Feng Tan, tanfeng@chinacdc.cn.

\footnotetext{
${ }^{1}$ Chinese Center for Disease Control and Prevention, Beijing, China; ${ }^{2}$ National Institute of Environmental Health, Chinese Center for Disease Control and Prevention, Beijing, China.

\& Joint first authors.
} 